\title{
Editorial
}

\section{Global and Regional Remote Sensing Precipitation Estimation, Evaluation, and Applications}

\author{
Youcun Qi $\mathbb{D}^{1},{ }^{1}$ Bin Yong, ${ }^{2}$ and Yalei You ${ }^{3}$ \\ ${ }^{1}$ Key Laboratory of Water Cycle and Related Land Surface Processes, \\ Institute of Geographic Sciences and Natural Resources Research, Chinese Academy of Sciences, Beijing, China \\ ${ }^{2}$ State Key Laboratory of Hydrology-Water Resources and Hydraulic Engineering, Hohai University, Nanjing 210098, China \\ ${ }^{3}$ Cooperative Institute for Climate and Satellites-Maryland, Earth System Science Interdisciplinary Center, \\ University of Maryland, College Park, MD, USA
}

Correspondence should be addressed to Youcun Qi; youcun.qi@igsnrr.ac.cn

Received 14 June 2018; Accepted 14 June 2018; Published 24 July 2018

Copyright (c) 2018 Youcun Qi et al. This is an open access article distributed under the Creative Commons Attribution License, which permits unrestricted use, distribution, and reproduction in any medium, provided the original work is properly cited.

In the past three decades, many precipitation products with high spatial-temporal resolutions have been developed. Global and regional quantitative precipitation estimations (QPEs) are very important for understanding climate variability and hydrometeorological cycles, improving flash flood and weather forecast, effectively managing the usage of earth's freshwater resources, detecting the natural disasters, and other hydrometeorological applications. However, obtaining accurate QPEs is a big challenge in many areas of the world due to sparse gauge networks and complicated terrains. Recent advances in remote sensing have allowed us to retrieve QPE information, representing one unprecedented contribution toward the global and regional mapping of precipitation.

Current global QPEs are often derived by combining the observations from both geostationary satellite (GEO) thermal infrared (IR) sensors and low-earth orbiting (LEO) satellite microwave imagers/sounders. Especially, the Tropical Rainfall Measuring Mission (TRMM) from late 1997 to early 2015, a joint mission between NASA and the Japan Aerospace EXploration Agency (JAXA), significantly improved the global QPEs. As the follow-on to TRMM, the Global Precipitation Measurement (GPM) Core Observatory satellite with a dual frequency precipitation radar (DPR) and a multichannel GPM microwave imager (GMI) was launched in February 2014, which provides great opportunity to further improve the global QPE development.

The GPM satellite constellation supports the next generation of unified global precipitation products with a better accuracy and a shorter data latency essential for researches and applications. The challenges faced in improving precipitation products are not only in developing better retrieval algorithms but also in improved approaches to integrate observations from the different sensors and then to assimilate them into various applications.

Furthermore, ground observations such as those from radar networks and gauge networks and disdrometer observations are available, providing potentially more accurate precipitation measurements on the ground than satellites. However, these observations are not available over oceans (except overseas adjacent to land). Thus, this special issue focused on improving precipitation products not only by developing newer retrieval algorithms but also through new approaches to integrate observations from the different sensors for assimilation into various applications.

Precipitation with high resolution and accuracy is of great significance for hydrological, meteorological, and ecological studies. Since satellite-based precipitation measurement is often too coarse for practical applications, it is crucial to develop spatial downscaling algorithms. Zhan et al. (2018) investigated two downscaling algorithms based on the multiple linear regression (MLR) and the geographically weighted regression (GWR), respectively. Both algorithms were applied to downscale annual and monthly precipitation obtained from the Global Precipitation Measurement (GPM) Mission in Hengduan Mountains, Southwestern China, from $10 \mathrm{~km} \times$ $10 \mathrm{~km}$ to $1 \mathrm{~km} \times 1 \mathrm{~km}$. These results indicated that GWR is a promising method in satellite precipitation downscaling researches and needed to be further studied. Sahlu et al. (2017) 
evaluated the performance of six satellite-based and three newly released reanalysis rainfall estimates at the daily timescale and the spatial grid size of 0.25 degrees during the period of 2000 to 2013 over the Upper Blue Nile Basin, Ethiopia, with the purpose of improving the reliability of precipitation estimates of the wet (June to September) and secondary rainy (March to May) seasons. They evaluated both adjusted and unadjusted satellite-based products of TMPA, CMORPH, PERSIANN, and ECMWF ERA-Interim reanalysis as well as Multi-Source Weighted-Ensemble Precipitation (MSWEP) estimates. Among the six satellite-based rainfall products, adjusted CMORPH exhibits the best accuracy of the wet season rainfall estimate. Overall, all precipitation datasets need further improvement in terms of detection during the occurrence of high rainfall intensity.

When the radar beam goes through the rain drops, the radar reflectivity usually suffers attenuation effects. Zhang et al. (2017) proposed a new method for attenuation correction of the radar reflectivity using the arbitrary oriented microwave link (referred henceforth to as ACML). Referring to the measurement of the arbitrary oriented microwave link, the ACML method optimizes the ratio of specific attenuation to specific differential phase which is a key parameter in attenuation correction schemes. The proposed method was evaluated using real data of a dual-polarization $\mathrm{X}$-band radar and measurements of two microwave links during two rainstorm events. The results showed that the variation range of the optimized ratio was overall consistent with the results of the previous studies. After attenuation correction with the optimal ratios, the radar reflectivity was significantly compensated, especially at the long distances. The corrected reflectivity was more intense than the reflectivity corrected by the "self-consistent" (SC) method and closer to the reflectivity of a nearby S-band radar. The effectiveness of the method was also verified by comparing the rain rates estimated by the X-band radar with those derived by rain gauges. It is demonstrated that the arbitrary oriented microwave link can be adopted to optimize the attenuation correction of radar reflectivity.

In order to improve the measurement of the precipitation microphysical characteristics sensor (PMCS), Liu et al. (2018) simulated the sampling process of raindrops by PMCS based on a particle-by-particle Monte Carlo model and discussed the effect of different bin sizes on DSD measurement. He also proposed the optimum sampling bin sizes for PMCS based on the simulation results. The simulation results of five sampling schemes of bin sizes in four rain-rate categories show that the raw capture DSD has a significant fluctuation variation influenced by the capture probability, whereas the appropriate sampling bin size and width can reduce the impact of variation of raindrop number on the DSD shape. The DSD obtained by PMCS and OTT has a good agreement.

Ice nuclei are a very important factor as they significantly affect the development and evolvement of convective clouds such as the hail clouds. Liu et al. (2017) conducted numerical simulations of hail processes in Zhejiang Province, China, using a mesoscale numerical model (WRF v3.4). The effects of six ice nuclei parameterization schemes on the macroscopic and microscopic structures of hail clouds were compared. The effect of the ice nuclei concentration on ground hailfall is stronger than on ground rainfall. There were significant spatiotemporal, intensity, and distribution differences in hailfall. Liu et al. (2018) run a numerical simulation of a southwest vortex rainstorm process. The results show that the low-pressure system originating from the Tibetan Plateau affects the southwest vortex mainly at the middle level, causing the strength increase of the southwest vortex (SWV), and acts as a connection between the positive vorticity centers at the upper and lower layers. As the ice crystals grow up, snow and graupel particles form, which substantially enhances the precipitation. This effect leads to the rapid development of SWV rainstorm clouds and the occurrence of precipitation. In addition to the effect of the plateau vortex, the subsequent merging of the convective clouds is another important factor for heavy rainfall because it also leads to development of convective clouds, causing heavy rainfall.

Yang et al. (2017) investigated the terrestrial water storage anomalies (TWSAs) in the Tarim River Basin (TRB) and analyzed the related factors of water variations in the mountain areas based on Gravity Recovery and Climate Experiment (GRACE) data with in situ river discharge and gauge observations during the period of 2002-2015. The results showed that three obvious flood events in 2005, 2006, and 2010 resulted in significant water surplus, although TWSA decreased in the TRB during 2002-2015. The significant water deficits were not well consistent with the negative anomalies of precipitation. While the river discharge behaved with low correlations with TWSA, linear relationships between TWSA and climate indices were insignificant in the TRB from 2002 to 2015.

Youcun Qi Bin Yong Yalei You 

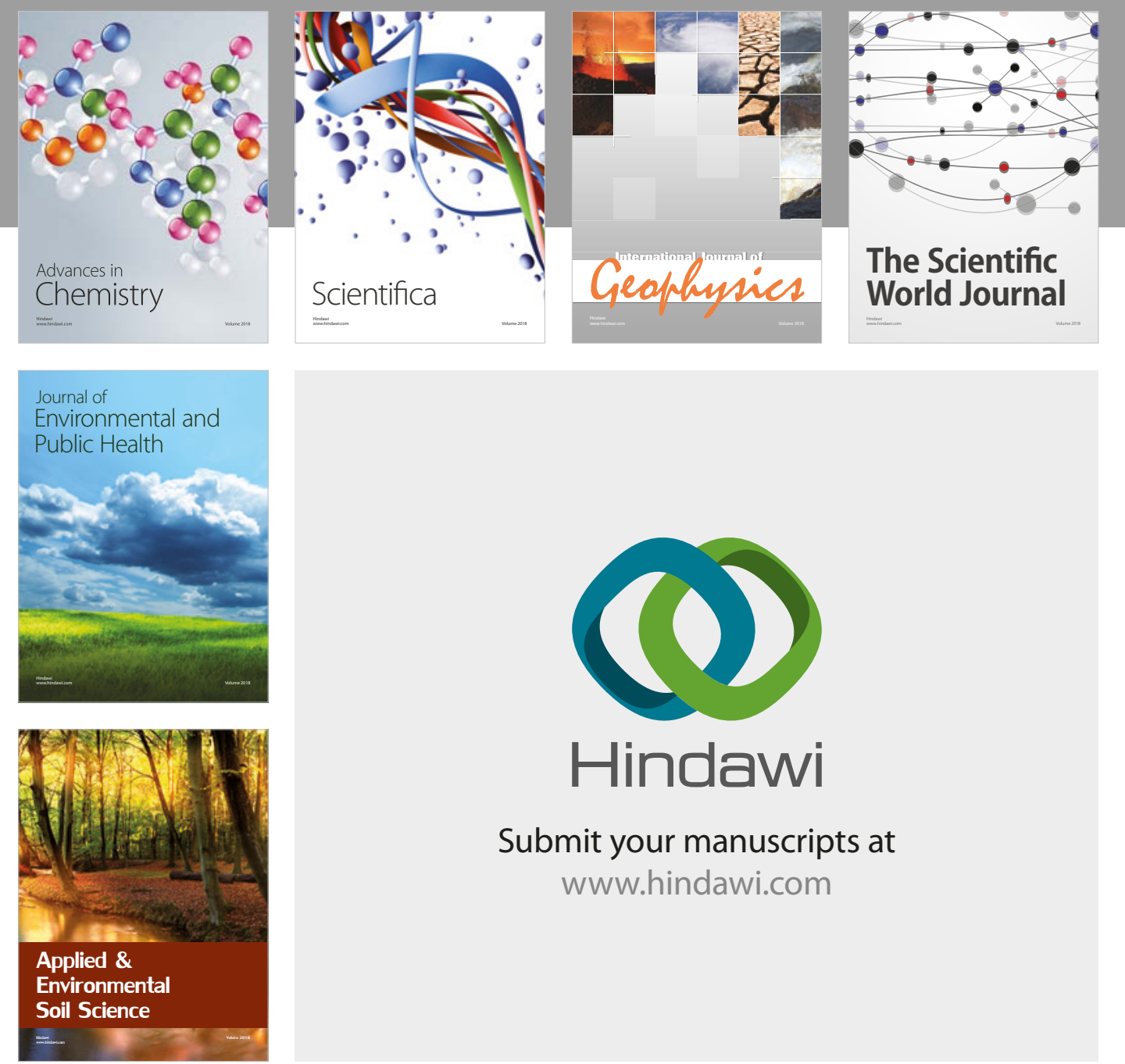

The Scientific

\section{World Journal}
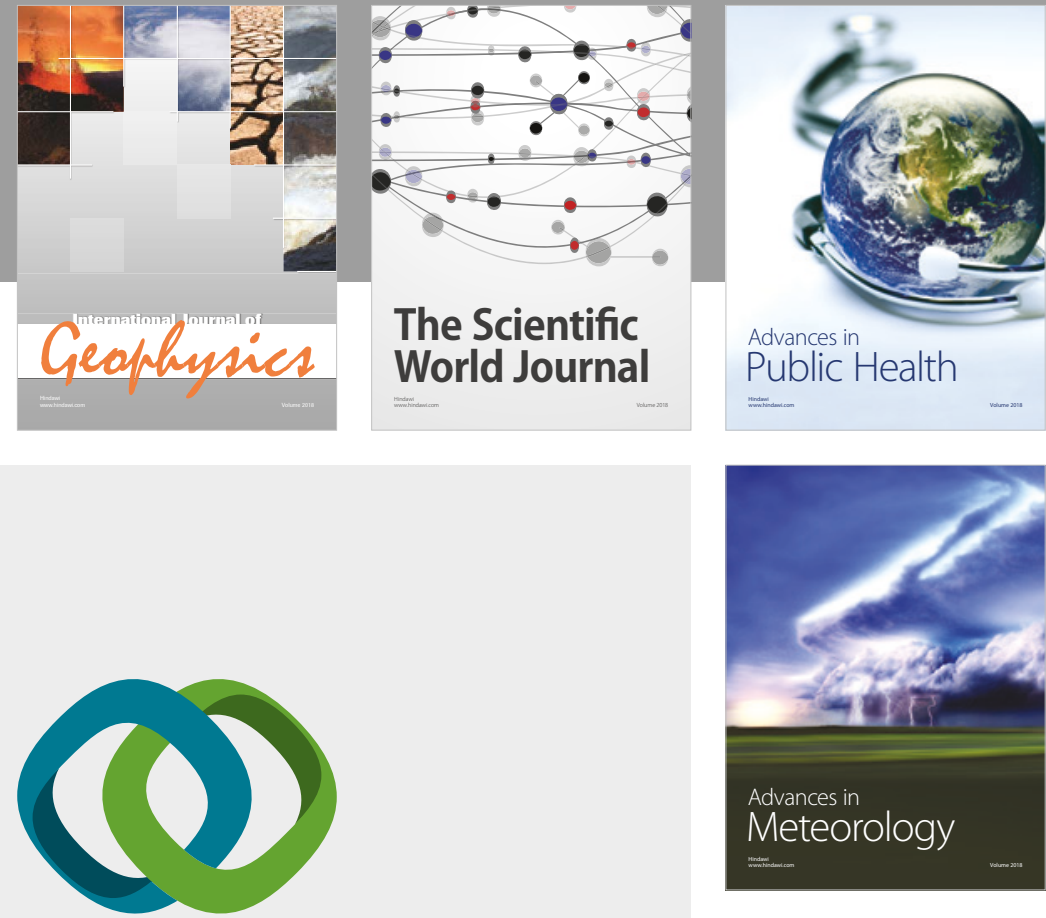

Advan

Public Health

\section{Hindawi}

Submit your manuscripts at

www.hindawi.com
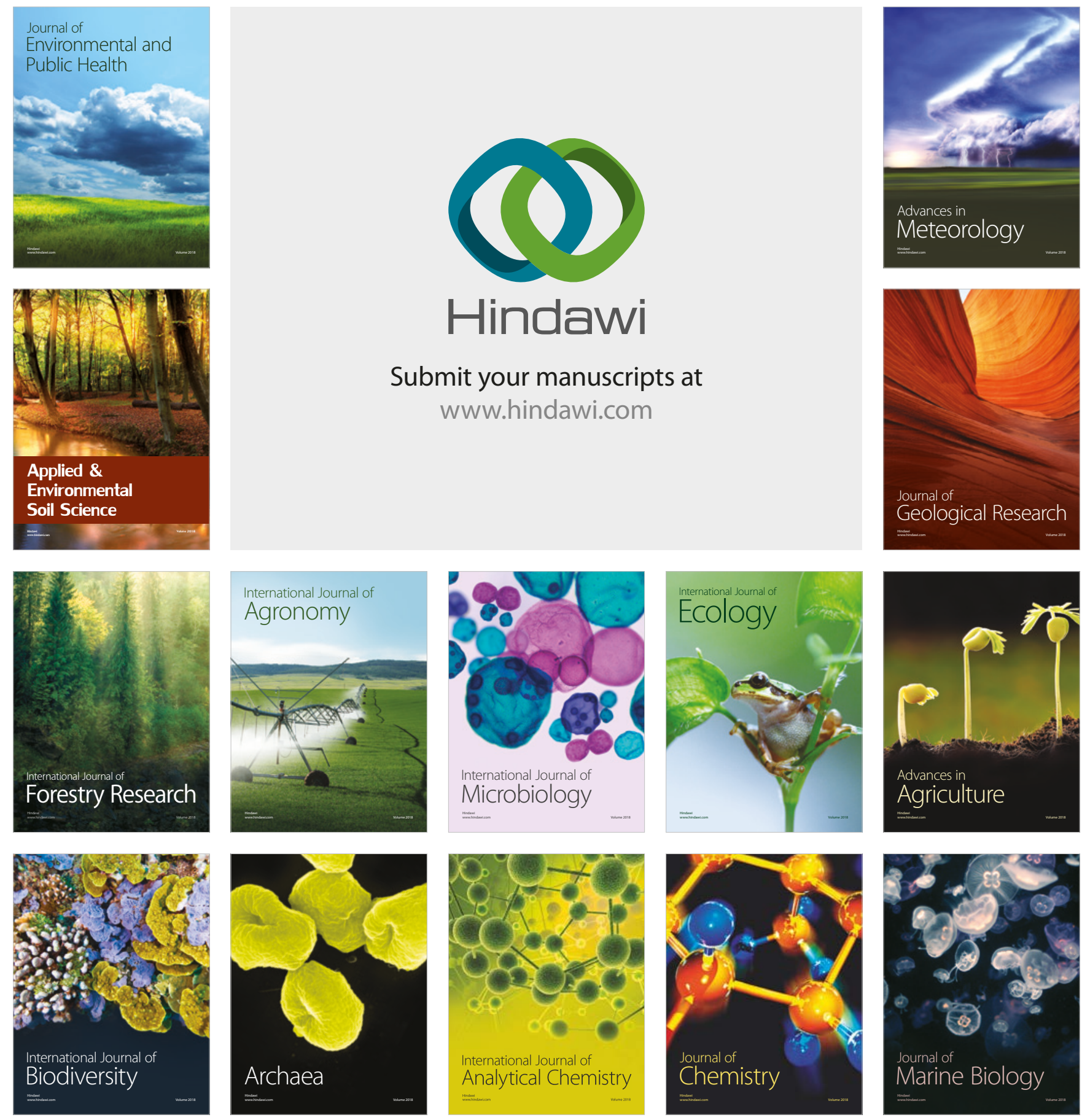